\title{
Cutting the cost of South African antiretroviral therapy using newer, safer drugs
}

W D F Venter, ${ }^{1}$ FCP (SA), MMed; B Kaiser ${ }^{2} \mathrm{MPH}$, PharmD, BCPS; Y Pillay, ${ }^{3} \mathrm{PhD} ;$ F Conradie, ${ }^{4} \mathrm{MB}$ BCh; G B Gomez,${ }^{5} \mathrm{PhD} ; \mathbf{P}$ Clayden $;{ }^{6}$ M Matsolo; $;$ C Amole, ${ }^{8}$ BA; L Rutter, ${ }^{7}$ BA; F Abdullah, ${ }^{9} \mathrm{MB}$ ChB, FCPHM, BSc Hons (Epi); E J Abrams, ${ }^{10}$ MD; C P Casas, ${ }^{11}$ MSc; M Barnhart, ${ }^{12} \mathrm{MD}$, MPH; A Pillay, ${ }^{13} \mathrm{PhD}$; A Pozniak,${ }^{14} \mathrm{MD}$, FRCP; A Hill,${ }^{14} \mathrm{PhD}$; L Fairlie, ${ }^{1}$ FCPaed (SA); M Boffito, ${ }^{14} \mathrm{MD}, \mathrm{PhD}$; M Moorhouse, ${ }^{1} \mathrm{MB}$ BCh; M Chersich, ${ }^{1} \mathrm{MB}$ BCh, PhD; C Serenata, ${ }^{1} \mathrm{MBA}$; J Quevedo, ${ }^{8}$ BS; G Loots ${ }^{15}$

${ }^{1}$ Wits Reproductive Health and HIV Institute, University of the Witwatersrand, Johannesburg, South Africa

${ }^{2}$ Formerly UNITAID, Geneva, Switzerland

${ }^{3}$ HIV/AIDS, TB and Maternal, Child and Women's Health in the South African National Department of Health, Pretoria, South Africa

${ }^{4}$ Clinical HIV Research Unit, University of the Witwatersrand and Southern African HIV Clinicians Society, Johannesburg, South Africa

${ }^{5}$ Amsterdam Institute for Global Health and Development, Department of Global Health, Academic Medical Center, Amsterdam, The Netherlands

${ }^{6} \mathrm{HIV}$ i-Base, London, UK

${ }^{7}$ Treatment Action Campaign, Cape Town, South Africa

${ }^{8}$ Clinton Health Access Initiative, New York, USA

${ }^{9}$ South African National AIDS Council, Pretoria, South Africa

${ }^{10}$ International Center for AIDS Care and Treatment Programs (ICAP), Mailman School of Public Health and College of Physicians and Surgeons, Columbia University, New York, USA

${ }^{1 "}$ UNITAID, Geneva, Switzerland

${ }^{12}$ USAID Bureau for Global Health, Washington, DC, USA

${ }^{13}$ Health Regulation and Compliance, South African National Department of Health, Pretoria, South Africa

${ }^{14}$ Chelsea and Westminster Hospital and St Stephens AIDS Trust, London, UK

${ }^{15}$ South African Department of Science and Technology, Pretoria, South Africa

Corresponding author: W D F Venter (fventer@wrhi.ac.za)

Antiretrovirals are a significant cost driver for HIV programmes. Current first-line regimens have performed well in real-life programmes, but have a low barrier to virological resistance and still carry toxicity that limits adherence. New drug developments may mean that we have access to safer, more robust and cheaper regimens, but only if the appropriate clinical trials are conducted. We briefly discuss these trials, and demonstrate the large cost savings to the South African HIV programme if these are successful.

S Afr Med J 2017;107(1):28-30. DOI:10.7196/SAMJ.2017.v107i1.12058

South Africa (SA) may be able to provide antiretroviral therapy (ART) to several million more people with HIV by 2019, using the current drug budget. While planned simplification of HIV service delivery promises to reduce expenditure, the drug cost of ART provision consumes the bulk of most HIV programme budgets, and is unlikely to decrease significantly, as multiple generic competitors have realised manufacturing efficiencies for current formulations. SA has the largest ART programme in the world, with domestic funding accounting for $>75 \%$ of all HIV spending. ${ }^{[1]}$ For the year $2014-2105$, USD350 million was spent on ART for just $<3$ million people living with HIV, most of it on first-line treatment. ${ }^{[2-4]}$ From mid-2016, the number of people on ART has increased to 3.4 million, 145000 of them on secondline treatment and $>700$ on third-line treatment. An increase in public spending is expected to continue, owing in part to recent changes in ART initiation guidelines. The national Department of Health (DoH) announced its endorsement to 'treat all' approaches from September 2016, dropping CD4 thresholds for ART initiation completely, theoretically doubling the number eligible for ART to $>6$ million people. ${ }^{[4]}$ This change and the steady improvement in life expectancy of patients on ART mean that the ART budget must accommodate millions of people for several decades of life. ${ }^{[5,6]}$
With $>300000$ people initiating therapy annually (a number that will almost double, according to the $\mathrm{DoH}$ ) on the efavirenz/tenofovir disoproxil fumarate/emtricitabine/ (EFV/TDF/FTC) fixed-dose generic (FTC and lamivudine (3TC) are interchangeable in terms of efficacy and cost, with 3TC used more in the private sector and outside $\mathrm{SA}^{[5]}$ ), SA is the largest consumer of generic ART in the world, using a quarter of global production. Two drugs, dolutegravir (DTG) and a new prodrug variant of tenofovir, tenofovir alafenamide (TAF), have been or are currently in the process of being licensed to multiple generic manufacturers by originator manufacturers directly or through voluntary licensing via Medicine Patent Pool (United Nations, Switzerland) processes. In short, replacing EFV, which is very vulnerable to resistance and drives most first-line side-effects, with DTG provides a better tolerated and cheaper drug with a high resistance barrier, limiting transition to expensive and less welltolerated, toxic next-line treatments. TAF is substantially cheaper than TDF, a major cost driver of first-line therapy, and provides some bone and renal toxicity benefits. ${ }^{[5,7]}$ Co-formulating the two drugs with FTC or 3TC would also substantially reduce the size of the current tablet, making it easier to manufacture, while saving on packaging and storage space. However, the two drugs - TAF and DTG - are manufactured by competing companies, where commercial 
Table 1. Summary of the ADVANCE study

Investigator driven, study drug donated by company

Funded by USAID and UNITAID

Conducted in Johannesburg, start date: beginning 2017

Non-inferiority design, 1 100 participants, over 96 weeks

Eligibility criteria

Needing first-line ART

No TB treatment, not pregnant (addressed in other studies; those contracting $\mathrm{TB}$ or falling pregnant may continue in the study)

No CD4 threshold

$>12$ years of age, $>40 \mathrm{~kg}$

Compares three combinations: DTG/TAF v. DTG/TDF v. EFV/ TDF (current first line in SA), all with FTC

Primary endpoint is viral suppression at 48 weeks

Pharmacokinetic sampling of both DTG/TAF in those who contract TB or become pregnant

USAID = United States Agency for International Development.

interests in high-income countries compel them to develop other co-formulations, which are of substantially higher cost or have significant questions regarding their use in patients with tuberculosis (TB) or in pregnancy. Botswana is planning a move to a DTG-based regimen for first line, based on the impressive performance of this regimen in high-income countries, but in the absence of data on $\mathrm{TB}$ and pregnancy.

A large, independent, randomised controlled study (ADVANCE) (Table 1), will address the substantial burden of evidence needed to change guidelines, with the hope that this study will provide evidence for initiating and switching millions of people on ART to the new regimen (to date, the combination DTG/TAF/FTC has not been specifically tested in clinical trials). Results from ADVANCE may be available by the end of 2018 for Medicines Control Council (MCC) registration and $\mathrm{DoH}$ guidelines and tender processes, so that the new regimen can be rolled out from 2019. The ADVANCE study has been designed by a unique multidisciplinary group of researchers, clinicians, activists, donors, and public health specialists, with substantial input and support from agencies, such as the World Health Organization (WHO) and the national DoH, as well as both originator and generic manufacturers. Aligned studies will address certain issues, such as the use of the new drugs in patients receiving rifampicin-containing $\mathrm{TB}$ regimens, and among pregnant women and adolescents, while partners are further working on new technologies, including nanotechnologies, to reduce the price of second-line drugs.

The introduction in SA of this new regimen has the potential to substantially reduce the cost of first-line ART, while also being safer and better tolerated, therefore limiting the need for second-line ART. We estimate potential cost savings for SA if the studies demonstrate efficacy and safety, and justify the urgency in completing the study swiftly and transitioning to the new combination without delay.

\section{Cost and other assumptions in the model}

Cost savings of introducing the new regimen can be expected from:

- Lower drug costs of DTG/TAF-containing regimens compared with current first-line drugs. The cost of current first-line therapy is $\sim$ USD110/patient/year. ${ }^{[3,6]}$ We have estimated an initial $20 \%$ saving (a conservative estimate provided to the authors by manufacturers for DTG/TAF cost saving over the current regimen of EFV/TDF/FTC) and a 50\% saving once volumes are met (as estimated by the Clinton Health Access Initiative (CHAI), USA).

- Limiting transition of patients from first- to second-line therapy owing to better tolerability and robustness of DTG/TAF-containing regimens. These savings are expected to accumulate through lower second-line drug costs (second-line therapy costs $\sim$ USD $350^{[5,8]}$ ), but also lower service delivery costs and decreased viral load monitoring.

The rate of transition from first-line to second-line regimens is not available. Since 2004, the number of SA patients on second-line treatment have increased to 145000 . These figures may have been initially inflated owing to the use of more toxic stavudine-containing regimens in use until 2010. In the calculations, we assume a $2 \%$ annual transition from first- to second-line treatment for anyone on the EFV-containing regimen ( $\mathrm{DoH}$ assumed $4 \%$ for the previous tender, but the limited data suggest that this is an underestimation). In comparison, as no DTG-related virological failure due to resistance has occurred in the initial 600000 patients on first-line treatment in Europe or the USA, we assumed that there would be no significant switching to second-line therapy in the years modelled ${ }^{[9]}$ For simplicity, and because the numbers are small, we have not factored in the effect on third-line initiations.

A move to DTG-TAF-based regimens will have to be phased as manufacturers scale-up and patients (both new initiations and established patients) transition to the new regimen. In this analysis, we compare three transition scenarios of different scale-up assumptions with the current status quo:

- Status quo: EFV/TDF/FTC remains the first-line regimen, with 300000 people initiating first-line treatment annually and $2 \%$ migrating to second-line treatment.

- Conservative scenario: continued initiation at 300000 annually at first only new initiations qualifying for DTG/TAF/FTC, others remaining on $\mathrm{EFV} / \mathrm{TDF} / \mathrm{FTC}$ and slowly transitioning to the new regimen over 3 years.

- Moderate scenario: initiations at 400000 annually, with at first only new initiations qualifying for DTG/TAF/FTC, and transitioning over 2 years.

- Aggressive scenario: initiations at 500000 annually, with everyone moving to DTG/TAF/FTC within a year.

Calculations are for expected savings in drug costs for 4 years from 2019; USD values are used, as manufacturing is largely dependent on USD-based acquisitions of materials. The USD450 million baseline is the estimated cost of ART in 2019, based on the current costs for 2014 - 2015 and the estimated programme growth. We note that there are currently wide currency fluctuations, introducing manufacturing and other uncertainties. Future costs have not been discounted, nor lost-to-follow-up costs or decreased costs associated with less second-line monitoring; we have also not factored in costs of the introduction of new regimens, such as training.

\section{Resullts}

As shown in Figs 1 and 2, all scenarios for the introduction of DTG/TAF-based regimens translate into substantial reductions in ART drug costs for SA. The aggressive model with new drugs treats 800000 people more than the status quo over 4 years, and with $>$ USD200 million saving. Moreover, using the current budget for ART drugs, we could cover the ART drug costs of treating an additional 2 million people. 


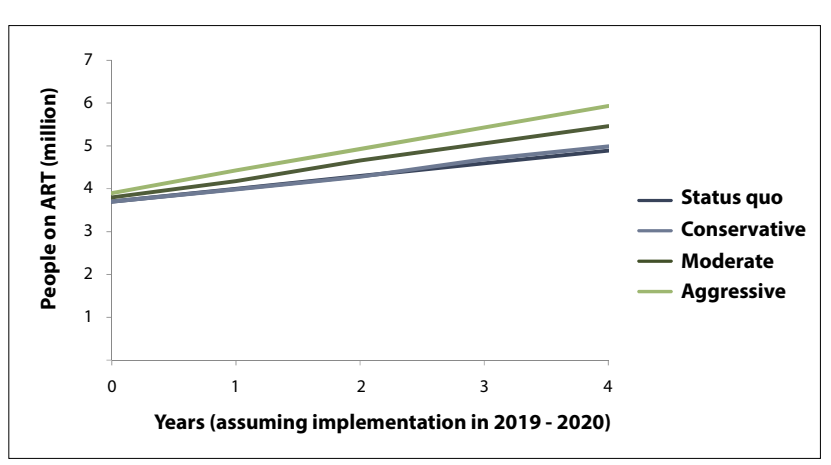

Fig. 1. Estimated increase in patients needing ART in SA. Number of people on antiretrovirals in SA (status quo $=300000$ initiating therapy annually, $2 \%$ migration to second line; conservative $=300000$ annually, transition from old regimen (EFV/TDF/FTC) to new (DTG/TAF/FTC) over 3 years; moderate $=$ new regimen, 400000 annually, transition over 2 years; aggressive $=$ new regimen, 500000 annually, transition over 1 year $)$.

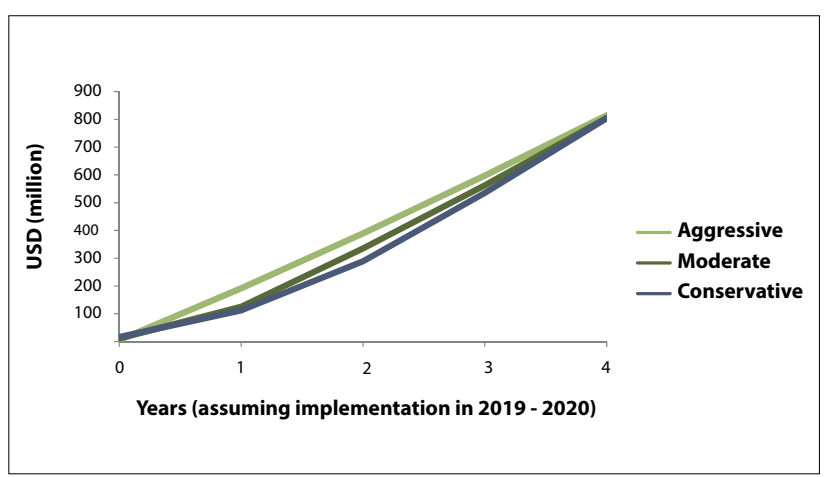

Fig. 2. Estimated crude savings on antiretroviral drugs (assuming implementation of new regimen in 2019 -2020). Cumulative savings compared with status quo (conservative $=300000$ annually, transition from old regimen to new over 3 years; moderate $=$ new regimen, 400000 annually, transition over 2 years; aggressive =new regimen, 500000 annually, transition over 1 year).

\section{Conclusion}

The introduction of DTG/TAF-based regimens could potentially translate into substantial savings to the SA health budget, if ADVANCE is completed rapidly and is successful, and if manufacturing is quickly scaled up to meet demand. Essentially, with the current ART budget, we could purchase DTG/TAF/FTC for everyone taking ART at the end of 4 years, even assuming the high end of 500000 new patients needing treatment annually.

Clinical evidence of the efficacy of both drugs is compelling, although questions remain around drug interactions with rifampicin and use in pregnancy, all being evaluated in clinical trials. In tandem, addressing the complex registration of new drugs (TAF has yet to be registered by the MCC) and combinations, including those of generic manufacturers, will need synchronisation, currently being co-ordinated locally by CHAI, in partnership with the DoH. If TAF is not registered in time, or if problems arise in the TB or pregnancy studies, the DTG/TAF/FTC regimen also being tested in ADVANCE confers significant clinical and cost benefits. Finally, ART drug costs account for about a quarter of cost of HIV care in SA (it is a greater proportion in countries where salaries are lower and laboratory monitoring is more limited); therefore, attention to systemic cost drivers are still needed. ${ }^{[7,10]}$

If successful, patients will benefit with a much safer and more forgiving regimen in a smaller tablet, government and other funders will save money, while pharmaceutical producers will have simplified manufacturing, using lower volumes of active pharmaceutical ingredients. In an age of escalating medical costs, we are seldom presented with a win-win scenario with regard to clinical care. The clinical studies and registration processes to secure inclusion of these two new drugs, if successful, would be a huge SA victory to complement the current successful ART roll-out, with knock-on effects for the entire region and other low- and middle-income countries.

Disclosure. Several authors are involved in the ADVANCE studies and sub-studies, with the support of the United States President's Emergency Plan for AIDS Relief (PEPFAR)/United States Agency for International Development (USAID) and UNITAID, in a broader collaboration addressing drug formulation and introduction, called OPTIMIZE. The study drug is donated by the originator companies, ViiV Healthcare (UK) and Gilead Sciences (USA). The views of this study are those of the authors and do not necessarily reflect the views of USAID, UNITAID or the SA or US governments. Raw data may be requested from the corresponding author, W D F Venter (fventer@wrhi.ac.za).

1. National Department of Health and South African National AIDS Council. South African HIV and TB investment case - summary report phase 1. 2016. http://sanac.org.za/wp-content/uploads/2016/03/1603Investment-Case-Report-LowRes-18-Marpdf (accessed 13 August 2016).

2. Surgey G, Cohen S, Guthrie T. Public health spending on antiretroviral medication in South Africa: An analysis of the annual ARV budget and expenditure for the financial year 2013/14, prepared for the South African National AIDS Council (in press).

3. South African Government News Agency. Tender to supply ARVs awarded to companies. 2015. http:// www.sanews.gov.za/south-africa/tender-supply-arvs-awarded-companies (accessed 17 September 2016). Centre for Health Journalism. Health Minister Aaron Motsoaledi admits that 'key leaders were in denial. 2016. http://bhekisisa.org/article/2016-07-15-aids2016-health-minister-aaron-motsoaledi-admitsthat-key-leaders-were-in-denial/ (accessed 27 July 2016).

5. Clinton Health Access Initiative. ARV market report: The state of the antiretroviral drug market in low- and middle-income countries, 2014 - 2019. 2015. http://www.clintonhealthaccess.org/content uploads/2015/11/CHAI-ARV-Market-Report-2015_FINAL.pdf (accessed 27 July 2016).

6. Sabin CA. Do people with HIV infection have a normal life expectancy in the era of combination antiretroviral therapy? BMC Med 2013;27(11):251. http://dx.doi.org/10.1186/1741-7015-11-251

7. Barnhart M, Shelton JD. ARVs: The next generation. Going boldly together to new frontiers of HIV treatment. Glob Health Sci Pract 2015;3(1):1-11. http://dx.doi.org/10.9745/GHSP-D-14-00243

8. Médecins Sans Frontières. Untangling the web of antiretroviral price reductions. http://issuu.com/ Médecins Sans Frontières. Untangling the web of antiretroviral price reductions. http://issuu.com//
msf_access/docs/hiv_report_untangling-the-web-18the/25? $=3239302 / 37230248$ (accessed 27 July 2016). 9. Brenner BG, Wainberg MA. Clinical benefit of dolutegravir in HIV-1 management related to the high Brenner BG, Wainberg MA. Clinical benefit of dolutegravir in HIV-1 management related to the high
genetic barrier to drug resistance. Virus Res 2016;pii:S0168-1702(16)30283-0. http://dx.doi.org/10.1016/j virusres.2016.07.006

10. Vu L, Waliggo S, Zieman B, et al. Annual cost of antiretroviral therapy among three service delivery models in Uganda. J Int AIDS Soc 2016;20(19)(5 Suppl 4):20840. http://dx.doi.org/10.7448/IAS.19.5.20840

Accepted 3 October 2016 